\title{
An atypical CRISPR-Cas locus in Symbiobacterium thermophilum flanked by a transposase, a reverse transcriptase, the endonuclease MutS2 and a putative Cas9-like protein Sandeep Chakraborty, R - 44/ 1, Celia Engineers, T. T. C Industrial Area, Rabale, Navi Mumbai, 400701, India.
}

\begin{abstract}
Clustered regularly interspaced short palindromic repeats (CRISPR) is a prokaryotic adaptive defense system that assimilates short sequences of invading genomes (spacers) within repeats, and uses nearby effector proteins (Cas), one of which is an endonuclease (Cas9), to cleave homologous nucleic acid during future infections from the same or closely related organisms. Here, a novel CRISPR locus with uncharacterized Cas proteins, is reported in Symbiobacterium thermophilum (Accid:NC_006177.1) around loc.1248561. Credence to this assertion is provided by four arguments. First, the presence of an exact repeat (CACGTGGGGTTCGGGTCGGACTG, 23 nucleotides) occurs eight times encompassing fragments about 83 nucleotides long. Second, comparison to a known CRISPR-Cas locus in the same organism (loc.355482) with an endonuclease Cas3 (WP_011194444.1, 729 aa) 10000 nt upstream shows the presence of a known MutS2 endonuclease (WP_011195247.1, 801 aa) in approximately the same distance in loc.1248561. Thirdly, and remarkably, an uncharacterized protein (1357 aa) long is uncannily close in length to known Cas9 proteins (1368 for Streptococcus pyogenes). Lastly, the presence of transposases and reverse transcriptase (RT) downstream of the repeat indicates this is one of an enigmatic RT-CRISPR locus, Also, the MutS2 endonuclease is not characterized as a CRISPR-endonuclease to the best of my knowledge. Interestingly, this locus was not among the four loci (three confirmed, one probable) reported by crisperfinder (http://crispr.i2bc.paris-saclay.fr/Server/), indicating that the search algorithm needs to be revisited. This finding begs the question - how many such CRISPR-Cas loci and Cas9-like proteins lie undiscovered within bacterial genomes?
\end{abstract}




\section{Introduction}

The seemingly innocuous discovery of clustered regularly interspaced short palindromic repeats (CRISPR) in bacteria [1] was later established as a prokaryotic adaptive defense system that memorizes short sequences of invading genomes (spacers) within repeats [2-4], and uses nearby effector proteins (Cas), one of which is an endonuclease (Cas9) [5-7], to cleave subsequent infections from the same organism [8,9].

The CRISPR-Cas system is divided into two classes - class 1 (90\%) and class 2 (10\%) - based on whether there are multiple effectors or a single effector, respectively [10,11]. The simplicity of the single effector class 2 CRISPR-Cas systems has fueled an incredible acceleration in gene-editing technologies that have raised prospects of human disease management [12-14], simultaneously raising serious ethical issues [15-17].

Technical limitations of the application of the CRISPR-Cas system for therapeutic purposes arise from the non-specificity of cleavage (off-targets) [18,19], with several methods available to verify such non-desirable cleavages [20,21]. Understanding the structural basis of target recognition in these Cas9 proteins [22] helps in generating Cas9 variants through amino-acid substitutions that moderate target site recognition and cleavage to minimize off-target cleavage [23]. It has recently been proposed that there are 'pre-existing humoral and cell-mediated adaptive immune responses to Cas9 in humans' [24]. In addition to generating Cas9 variants that do no elicit immune response, unearthing novel Cas9 proteins could be another strategy to circumvent this problem.

Bacterial sequence diversity is mind-boggling. Even in reasonably well-characterized species, like Symbiobacterium thermophilum [25,26], there are many proteins that are uncharacterized. Could it be that some of the proteins are CRISPR-Cas related? Here, the genome of S. thermophilum has been analyzed for such proteins.

\section{Results and discussion}

There are four reasons that strongly support the assertion that loc.1248561 is a novel CRISPR-Cas locus.

1. An exact repeat - CACGTGGGGTTCGGGTCGGACTG, 23 nucleotides - occurs eight times, and has integrated seven fragments that are $\sim 83$ nucleotides long (Fig 1a), and is predicted (RNAfold [27]) to have the proper folding to ensure cleavage.

2. A known CRISPR-Cas locus in the same organism (loc.355482) has a 729 aa endonuclease CAS3 (WP_011194444.1) at 9440 nt from the repeat location (Table 1). Analogously, loc.1248561 has a 801 aa endonuclease (MutS2) 10819 nt away from the repeat location.

3. An uncharacterized protein (1357 aa) long located between the MutS2 endonuclease and the repeats is uncannily close in length to known Cas9 proteins (1368 aa for Streptococcus pyogenes).

4. The presence of a reverse transcriptase (RT, WP_011194877.1) downstream of the repeat indicates this locus is a RT-CRISPR that integrates RNA (after the RT transcribes it into DNA) [28-30], possibly using a proximal transposase $[31,32]$.

\section{The 23 nt repeats:}

The CRISPR repeats are typically varying in size from 23 to 47nt [33], which are transcribed and cleaved into mature CRISPR RNAs (crRNA) [34]. Although, some CRISPR-Cas loci have an trans-activating CRISPR RNA molecule (tracrRNA) that co-purifies with Cas9, and is essential for DNA interference [35], the locus here seems to have has no such sequence (the tracrRNA is homologous, but not exact to the crna). The secondary structure of the crna (Fig 1b) predicted by RNAfold [27] shows analogous folding to known repeats that allows cleavage [36].

Interestingly, this locus was not among the four loci (three confirmed, one probable) reported by Crisperfinder [33]. PILER-CR also did not detect this locus [37]. CRISPRdigger [38] and CRT [39] have not been evaluated. 
Crisperfinder has several criteria for choosing repeats and spacers, and 'and the percentage of identity between spacers is not allowed to exceed 60\%'. The presence of related protein in the vicinity of repeats (as well as it folding properties) are much stronger criteria, that would override such homology thresholds. This indicates that those parameters need to be revisited.

\section{MutS2 endonuclease}

To the best of my knowledge, MutS2 is not characterized as a CRISPR-endonuclease. MutS2 from Thermus thermophilus was found to suppress homologous recombination [40], whereas a recent study in Bacillus subtilis demonstrated the opposite phenomenon [41]. Also, though MutS2 was mentioned in the protocol for 'CRISPR/Cas9 Editing of the Bacillus subtilis Genome', there is no indication that its CRISPR-like endonuclease activity was discussed. Another study emphasized the requirement of all domains of this protein for its functionality [42]. The considerable sequence homology to other MutS2 proteins expectedly translates into significant structural homology (Fig 2a) on a predicted structures, and will help in identifying domains/active sites [43] and other promiscuous functions, if they exist [44].

\section{Reverse transcriptase based CRISPR loci}

The reverse flow of genetic information from RNA to DNA, essential to the retroviral replication cycle [45], was a momentous discovery (made independently by Temin and Baltimore in 1970) challenging Crick's famous 'central dogma' [46] that information flowed from DNA to RNA to proteins, and never in the reverse direction. These enzymes were identified in bacteria almost two decades later [47]. In many Type III CRISPR systems, a reverse transcriptase (RT) is fused to a Cas1, which in the marine bacterium Marinomonas mediterranea, was shown to mediate the in vivo acquisition of RNA spacers [28]. Two recent studies looked at the evolutionary origins of RT-CRISPR, and looked mostly at RT-Cas1 fusions [29,30]. Moreover, these work seeded on known CRISPR-Cas loci, and thus would not have looked at the locus described here. The spacer sequences here (Fig 1a) could not identify any known organism, echoing the observation that 'the vast majority of RT-linked CRISPR spacers come from a distinct sequence pool, the nature of which remains enigmatic' [29].

\section{Putative Cas9-like protein}

Another fascinating observation is the presence of an uncharacterized open reading frame (ORF, 1357 aa long), which is uncannily close to the size of known Cas9 proteins (1368 for Streptococcus pyogenes). Due to the lack of homology with known Cas9, the structure prediction (using SWISS-MODEL [48]) did not result in any realistic structure (Fig 2b), in contrast to the the MutS2. There is another long ORF (>200 aa) between the MutS2 endonuclease and the repeat location, and three such ORFs after the repeat location. These are all uncharacterized, even on a complete BLAST [49] to the nr database.

\section{Conclusion and future work:}

Seminal work on the enumeration and classification of CRISP-Cas systems first used the highly conserved Cas1 as the seed [10], and followed it up by using known CRISP-Cas sequences from databases [11] in an iterative search method. However, this underestimates bacterial genome diversity - and a completely novel CRISPR locus will escape detection. On, the other hand using repeated sequences as the starting point, and trying to look for relevant proteins (endonucleases, transposases, reverse transcriptases) could identify more CRISPR-Cas loci, and related Cas proteins. Even, the presence of uncharacterized protein coding open reading frames of reasonable length and number should suffice to tag a repeat as a putative CRISPR-Cas loci. Future work will build such a database. 


\section{Materials and methods}

ftp://ftp.ncbi.nih.gov/genomes/refseq/bacteria/Symbiobacterium_thermophilum/latest_assembly_versions/ provided the Symbiobacterium thermophilum genome and annotation. The getorf program from the EMBOSS suite [13] was used to obtain the ORFs (SI ORFS.fa). ORFs that are 15000 nt from the loci are annotated on both sides using YeATS [50-52] based on the Ncbi annotation.

RNAfold [27] was used to predict the secondary structure of the RNA repeat. Structures of the MutS2 and the novel predicted Cas9 were predicted using SWISS-MODEL [48]. Structures were superimposed using MUSTANG [53]. MSA figures were generated using the ENDscript server [54]. Protein structures were rendered by PyMol (http://www.pymol.org/).

\section{Competing interests}

No competing interests were disclosed.

\section{References}

1. Ishino Y, Shinagawa H, Makino K, Amemura M, Nakata A (1987) Nucleotide sequence of the iap gene, responsible for alkaline phosphatase isozyme conversion in escherichia coli, and identification of the gene product. Journal of bacteriology 169: 5429-5433.

2. Mojica FJ, García-Martínez J, Soria E, et al. (2005) Intervening sequences of regularly spaced prokaryotic repeats derive from foreign genetic elements. Journal of molecular evolution 60: 174-182.

3. Bolotin A, Quinquis B, Sorokin A, Ehrlich SD (2005) Clustered regularly interspaced short palindrome repeats (crisprs) have spacers of extrachromosomal origin. Microbiology 151: 2551-2561.

4. Horvath P, Barrangou R (2010) Crispr/cas, the immune system of bacteria and archaea. Science 327: $167-170$.

5. Barrangou R, Fremaux C, Deveau H, Richards M, Boyaval P, et al. (2007) Crispr provides acquired resistance against viruses in prokaryotes. Science 315: 1709-1712.

6. Jinek M, Chylinski K, Fonfara I, Hauer M, Doudna JA, et al. (2012) A programmable dual-rna-guided dna endonuclease in adaptive bacterial immunity. Science 337: 816-821.

7. Mali P, Yang L, Esvelt KM, Aach J, Guell M, et al. (2013) Rna-guided human genome engineering via cas9. Science 339: 823-826.

8. Marraffini LA (2015) Crispr-cas immunity in prokaryotes. Nature 526: 55-61.

9. Sander JD, Joung JK (2014) Crispr-cas systems for editing, regulating and targeting genomes. Nature biotechnology 32: 347-355.

10. Makarova KS, Wolf YI, Alkhnbashi OS, Costa F, Shah SA, et al. (2015) An updated evolutionary classification of crispr-cas systems. Nature Reviews Microbiology 13: 722-736.

11. Shmakov S, Smargon A, Scott D, Cox D, Pyzocha N, et al. (2017) Diversity and evolution of class 2 crispr-cas systems. Nature Reviews Microbiology 15: 169-182.

12. Cai L, Fisher AL, Huang H, Xie Z (2016) Crispr-mediated genome editing and human diseases. Genes \& Diseases 3: 244-251. 
13. Ma H, Marti-Gutierrez N, Park SW, Wu J, Lee Y, et al. (2017) Correction of a pathogenic gene mutation in human embryos. Nature 548: 413-419.

14. Liang P, Xu Y, Zhang X, Ding C, Huang R, et al. (2015) Crispr/cas9-mediated gene editing in human tripronuclear zygotes. Protein \& cell 6: 363-372.

15. Lanphier E, Urnov F (2015) Don't edit the human germ line. Nature 519: 410.

16. Nagatani RA, Gonzalez A, Shoichet BK, Brinen LS, Babbitt PC (2007) Stability for function trade-offs in the enolase superfamily "catalytic module". Biochemistry 46: 6688-6695.

17. Evitt NH, Mascharak S, Altman RB (2015) Human germline crispr-cas modification: toward a regulatory framework. The American Journal of Bioethics 15: 25-29.

18. Fu Y, Foden JA, Khayter C, Maeder ML, Reyon D, et al. (2013) High-frequency off-target mutagenesis induced by crispr-cas nucleases in human cells. Nature biotechnology 31: 822-826.

19. Zhang XH, Tee LY, Wang XG, Huang QS, Yang SH (2015) Off-target effects in crispr/cas9-mediated genome engineering. Molecular Therapy-Nucleic Acids 4: e264.

20. Park J, Childs L, Kim D, Hwang GH, Kim S, et al. (2017) Digenome-seq web tool for profiling crispr specificity. Nature Methods 14: 548-549.

21. Tsai SQ, Nguyen NT, Malagon-Lopez J, Topkar VV, Aryee MJ, et al. (2017) Circle-seq: a highly sensitive in vitro screen for genome-wide crispr-cas9 nuclease off-targets. Nature methods .

22. Anders C, Niewoehner O, Duerst A, Jinek M (2014) Structural basis of pam-dependent target dna recognition by the cas9 endonuclease. Nature 513: 569-573.

23. Chen JS, Dagdas YS, Kleinstiver BP, Welch MM, Sousa AA, et al. (2017) Enhanced proofreading governs crispr-cas9 targeting accuracy. Nature 550: 407.

24. Charlesworth CT, Deshpande PS, Dever DP, Dejene B, Gomez-Ospina N, et al. (2018) Identification of pre-existing adaptive immunity to cas9 proteins in humans. bioRxiv .

25. Ohno M, Shiratori H, Park MJ, Saitoh Y, Kumon Y, et al. (2000) Symbiobacterium thermophilum gen. nov., sp. nov., a symbiotic thermophile that depends on co-culture with a bacillus strain for growth. International journal of systematic and evolutionary microbiology 50: 1829-1832.

26. Ueda K, Yamashita A, Ishikawa J, Shimada M, Watsuji To, et al. (2004) Genome sequence of symbiobacterium thermophilum, an uncultivable bacterium that depends on microbial commensalism. Nucleic Acids Research 32: 4937-4944.

27. Mathews DH, Disney MD, Childs JL, Schroeder SJ, Zuker M, et al. (2004) Incorporating chemical modification constraints into a dynamic programming algorithm for prediction of rna secondary structure. Proceedings of the National Academy of Sciences of the United States of America 101: $7287-7292$.

28. Silas S, Mohr G, Sidote DJ, Markham LM, Sanchez-Amat A, et al. (2016) Direct crispr spacer acquisition from rna by a natural reverse transcriptase-cas1 fusion protein. Science 351: aad4234.

29. Silas S, Makarova KS, Shmakov S, Páez-Espino D, Mohr G, et al. (2017) On the origin of reverse transcriptase-using crispr-cas systems and their hyperdiverse, enigmatic spacer repertoires. MBio 8: e00897-17.

30. Toro N, Martínez-Abarca F, González-Delgado A (2017) The reverse transcriptases associated with crispr-cas systems. Scientific reports 7: 7089. 
31. Heffron F, McCarthy BJ, Ohtsubo H, Ohtsubo E (1979) Dna sequence analysis of the transposon tn3: three genes and three sites involved in transposition of tn3. Cell 18: 1153-1163.

32. Hickman AB, Dyda F (2014) Crispr-cas immunity and mobile dna: a new superfamily of dna transposons encoding a cas1 endonuclease. Mobile DNA 5: 23.

33. Grissa I, Vergnaud G, Pourcel C (2007) Crisprfinder: a web tool to identify clustered regularly interspaced short palindromic repeats. Nucleic acids research 35: W52-W57.

34. Deltcheva E, Chylinski K, Sharma CM, Gonzales K, Chao Y, et al. (2011) Crispr rna maturation by trans-encoded small rna and host factor rnase iii. Nature 471: 602-607.

35. Karvelis T, Gasiunas G, Miksys A, Barrangou R, Horvath P, et al. (2013) crrna and tracrrna guide cas9-mediated dna interference in streptococcus thermophilus. RNA biology 10: 841-851.

36. Sashital DG, Jinek M, Doudna JA (2011) An rna-induced conformational change required for crispr rna cleavage by the endoribonuclease cse3. Nature structural \& molecular biology 18: 680-687.

37. Edgar RC (2007) Piler-cr: fast and accurate identification of crispr repeats. BMC bioinformatics 8: 18.

38. Ge R, Mai G, Wang P, Zhou M, Luo Y, et al. (2016) Crisprdigger: detecting crisprs with better direct repeat annotations. Scientific reports 6: 32942.

39. Bland C, Ramsey TL, Sabree F, Lowe M, Brown K, et al. (2007) Crispr recognition tool (crt): a tool for automatic detection of clustered regularly interspaced palindromic repeats. BMC bioinformatics 8: 209 .

40. Fukui K, Nakagawa N, Kitamura Y, Nishida Y, Masui R, et al. (2008) Crystal structure of muts2 endonuclease domain and the mechanism of homologous recombination suppression. Journal of Biological Chemistry 283: 33417-33427.

41. Burby PE, Simmons LA (2017) Muts2 promotes homologous recombination in bacillus subtilis. Journal of bacteriology 199: e00682-16.

42. Wang G, Maier RJ (2017) Molecular basis for the functions of a bacterial muts2 in dna repair and recombination. DNA repair 57: 161-170.

43. Chakraborty S, Minda R, Salaye L, Bhattacharjee SK, Rao BJ (2011) Active site detection by spatial conformity and electrostatic analysis - unravelling a proteolytic function in shrimp alkaline phosphatase. PLoS ONE 6: e28470.

44. Chakraborty S, Rao BJ (2012) A measure of the promiscuity of proteins and characteristics of residues in the vicinity of the catalytic site that regulate promiscuity. PLoS ONE 7: e32011.

45. Telesnitsky A, Goff S (1997) Reverse transcriptase and the generation of retroviral dna .

46. Hahn FE (1973) Reverse transcription and the central dogma. In: Progress in Molecular and Subcellular Biology 3, Springer. pp. 1-14.

47. Lampson BC, Sun J, Hsu MY, Vallejo-Ramirez J, Inouye S, et al. (1989) Reverse transcriptase in a clinical strain of escherichia coli: production of branched rna-linked msdna. Science(Washington) 243: $1033-1038$.

48. Arnold K, Bordoli L, Kopp J, Schwede T (2006) The SWISS-MODEL workspace: a web-based environment for protein structure homology modelling. Bioinformatics 22: 195-201. 
49. Altschul SF, Madden TL, Schaffer AA, Zhang J, Zhang Z, et al. (1997) Gapped BLAST and PSIBLAST: a new generation of protein database search programs. Nucleic Acids Res 25: 3389-3402.

50. Chakraborty S, Britton M, Wegrzyn J, Butterfield T, Martinez-Garcia PJ, et al. (2015). YeATS-a tool suite for analyzing RNA-seq derived transcriptome identifies a highly transcribed putative extensin in heartwood/sapwood transition zone in black walnut.

51. Chakraborty S, Britton M, Martínez-García P, Dandekar AM (2016) Deep RNA-seq profile reveals biodiversity, plant-microbe interactions and a large family of NBS-LRR resistance genes in walnut (juglans regia) tissues. AMB Express 6: 1.

52. Martínez-García PJ, Crepeau MW, Puiu D, Gonzalez-Ibeas D, Whalen J, et al. (2016) The walnut (juglans regia) genome sequence reveals diversity in genes coding for the biosynthesis of nonstructural polyphenols. The Plant Journal .

53. Konagurthu AS, Whisstock JC, Stuckey PJ, Lesk AM (2006) MUSTANG: a multiple structural alignment algorithm. Proteins 64: 559-574.

54. Robert X, Gouet P (2014) Deciphering key features in protein structures with the new endscript server. Nucleic acids research 42: W320-W324.

55. Groothuizen FS, Winkler I, Cristóvão M, Fish A, Winterwerp HH, et al. (2015) Muts/mutl crystal structure reveals that the muts sliding clamp loads mutl onto dna. Elife 4: e06744.

56. Nishimasu H, Ran FA, Hsu PD, Konermann S, Shehata SI, et al. (2014) Crystal structure of cas9 in complex with guide rna and target dna. Cell 156: 935-949. 
bioRxiv preprint doi: https:/doi org/10.1101/252296; this version posted January 27, 2018. The copyright holder for this preprint (which was not certified by peer review) is the author/funder, who has granted bioRxiv a license to display the preprint in perpetuity. It is made available under aCC-BY-NC-ND 4.0 International license. 
Table 1: Annotation of open-reading frames (ORF) around the known CRISPR-loci (loc.355482, KNOWN) and the new CRISPR-loci (loc.1248561, NEW) in Symbiobacterium thermophilum: ORF numbering is based on the ORFs obtained using 'getorf' (SI ORFS.fa). ORFs that are 15000 nt from the loci are annotated on both sides (pre and post). The distance (9440) of the helicase/endonuclease CAS3 (WP_011194444.1, 729 aa) in loc.355482 to the repeats is almost the same as the distance (10819) of MutS2 endonuclease (WP_011195247.1, 801) to the repeats in loc.1248561. None of the apparently bona-fide proteins between the MutS2 and the repeats, some having ORFs as long as 1357 aa, are characterized. Intriguingly, the 1357 is uncannily close to length of known Cas9 proteins (1368 for Streptococcus pyogenes) - Symbiobacterium thermophilum does not have a Cas9 CRISPR-Cas locus. Finally, a group II intron reverse transcriptase (RT) downstream of the repeat suggest that this locus is from a family of enigmatic RT-CRISPR systems [29], while the locus also has transposases that explain the CRISPR-Cas genesis.

\begin{tabular}{|c|c|c|c|c|c|}
\hline Dir & ORF & LEN & Annotation & Start & End \\
\hline \hline \multirow{5}{*}{ KNOWN-pre } & 2548 & 703 & $?$ & 340008 & 342116 \\
& 2559 & 444 & IS110 family transposase & 342291 & 343622 \\
& 2590 & 442 & IS256 family transposase & 345631 & 346956 \\
& 2611 & 729 & CAS helicase/endonuclease CAS3 & 347066 & 349252 \\
& 2633 & 625 & TYPE I-C CAS8C/CSD1 & 349868 & 351742 \\
& 2640 & 385 & TYPE I-C CAS7/CSD2 & 351697 & 352851 \\
& 2656 & 392 & IS5/IS1182 family transposase & 354046 & 355221 \\
\hline & & & Repeat location 355482 & & \\
\hline \multirow{3}{*}{ KNOWN-post } & 2689 & 683 & $?$ & 357654 & 359702 \\
& 2739 & 695 & Hypothetical protein & 363051 & 365135 \\
& 2748 & 308 & Hypothetical protein & 365125 & 366048 \\
& 2774 & 302 & Hypothetical protein & 368033 & 368938 \\
\hline \hline
\end{tabular}

\begin{tabular}{|c|c|c|c|c|c|}
\hline \hline \hline NEW-pre & 8862 & 646 & ABC Transporter ATP-binding protein & 1234429 & 1236384 \\
& 8867 & 211 & DUF421 domain-containing protein & 1235825 & 1237183 \\
& 8871 & 320 & Hypothetical protein & 1236972 & 1238081 \\
& 8886 & 801 & Endonuclease MutS2 & 1238171 & 1240573 \\
& 8902 & 215 & $?$ & 1242464 & 1243420 \\
& 8913 & 1357 & $?$ (Putative Cas9) & 1240660 & 1244943 \\
\hline & & & Repeat location 1248561 & & \\
\hline \multirow{5}{*}{ NEW-post } & 8950 & 305 & $?$ & 1249228 & 1250463 \\
& 8974 & 264 & Tyrosine-trna ligase & 1252673 & 1253659 \\
& 8981 & 409 & $?$ & 1253070 & 1254419 \\
& 8988 & 325 & Group II intron Reverse transcriptase/maturase & 1254535 & 1255509 \\
& 9007 & 611 & Adenylyltransferase & 1257668 & 1257593 \\
& 9015 & 254 & Hypothetical protein & 1258183 & 1259477 \\
& 9020 & 269 & Transposase & 1259556 & 1261148 \\
& 9033 & 530 & Transposase & 1262612 & 1263895 \\
\hline
\end{tabular}



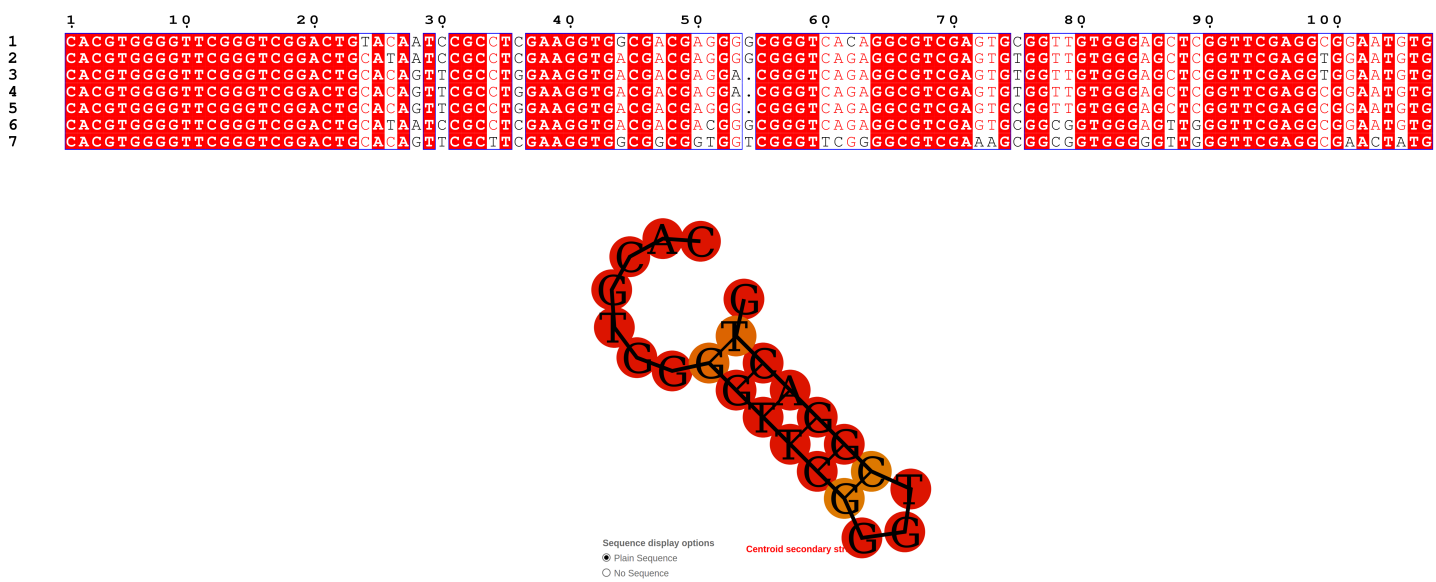

Figure 1: Repeats and spacers in the new locus: The sequence CACGTGGGGTTCGGGTCGGACTG (23 nt) is repeated eight times, and encompasses seven fragments about 83 nucleotides long. The secondary structure of the crna is predicted by RNAfold [27]. Note there two mismatched pairs (G-T). The cleavage site is predicted to the sequence at the end of the stem on the 3' end [36]. 

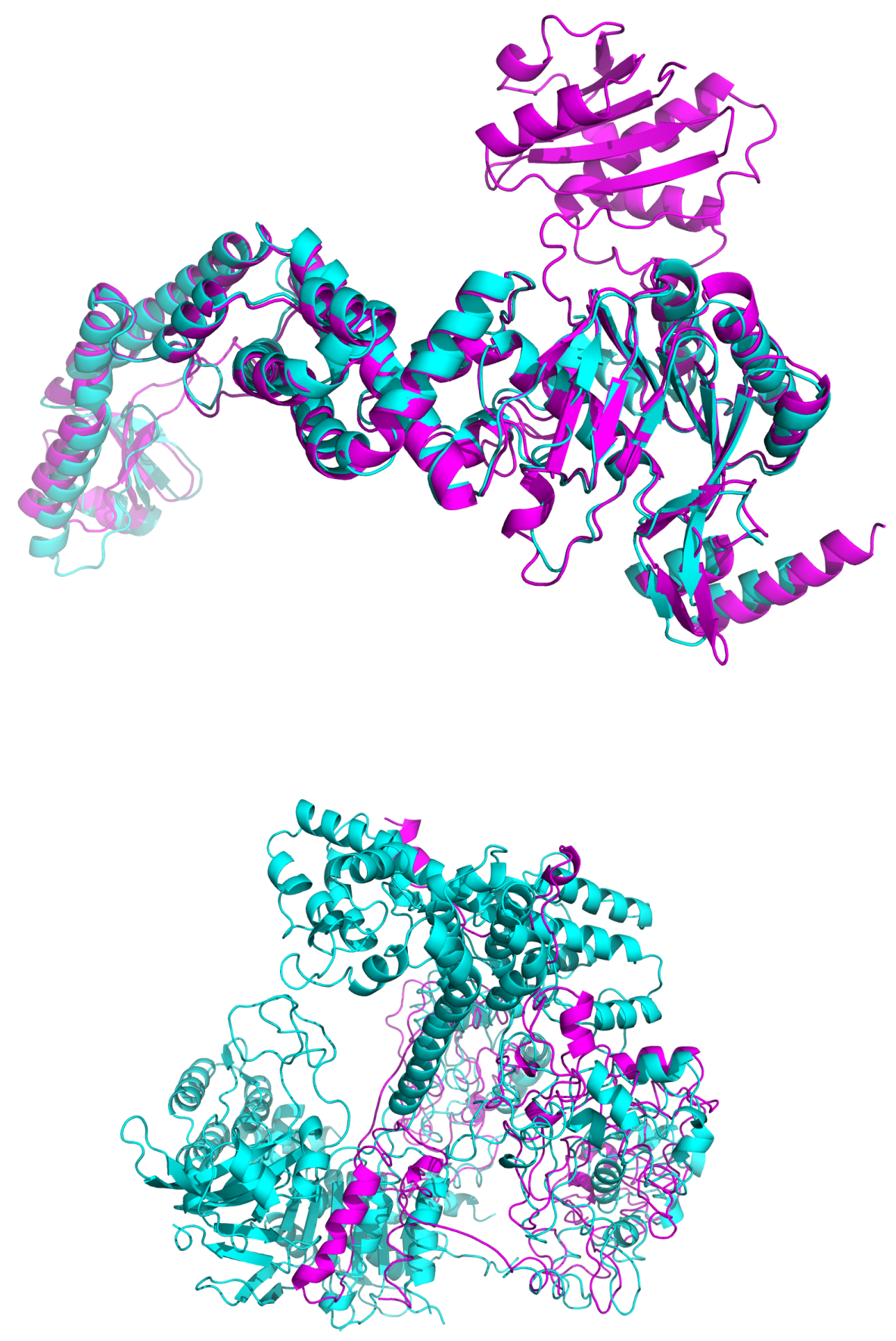

Figure 2: Structure prediction and superimposition for Muts2 and the novel Cas9: (a) Structure of Muts2 (cyan) predicted by SWISSMODEL using PDBid:5AKC (magenta) ('MutS in complex with the Nterminal domain of MutL' from Escherichia coli [55]) as template. The superimposition shows good structural homology. (b) Structure of novel Cas9 (magenta) predicted by SWISSMODEL using PDBid:4OO8 (cyan) ('Crystal structure of Streptococcus pyogenes Cas9 in complex with guide RNA and target DNA' [56]) as template. Due to lack of sequence homology, the structure prediction method does not predict a reasonable structure. Future functional and structural characterization of this putative Cas9 will reveal these features. 